\title{
Tireur olympique/embusqué : récit sportif et fiction de la guerre dans Robert Mitchum ne revient pas de Jean Hatzfeld
}

\author{
Pierre VAUCHER \\ Université Laval/Université de Gand \\ Québec, Canada/Belgique
}

\begin{abstract}
Résumé : Notre intérêt porte sur les rapports entre sport et guerre dans la littérature, qui forcent à questionner cette forme assumée de la violence qu'est l'engagement armé. L'analyse s'appuie sur un roman de Jean Hatzfeld, intitulé Robert Mitchum ne revient pas, et qui prend pour cadre la guerre de Bosnie-Herzégovine. En mêlant sport, guerre et journalisme, l'auteur pose les enjeux du témoignage de la guerre entre fascination pour la lutte et pacifisme.
\end{abstract}

\begin{abstract}
We would like to focus on the relationship between sport and war in literature by interrogating military engagement as an accepted form of violence. To address this issue, we analyse a narrative fiction by Jean Hatzfeld, entitled Robert Mitchum ne revient pas [Robert Mitchum is not coming back], which is based on the Bosnian War. By mixing sport, war and journalism, the author discusses the challenges of war testimony, between fascination with violence and pacifism.
\end{abstract}

Mots-clés : guerre de Bosnie-Herzégovine, récit de guerre, sport, journalisme, témoignage.

Keywords : Bosnian War, war narrative, sport, journalism, testimony.

\section{Introduction}

Jean Hatzfeld publie, en 2013, un roman dont l'action met en scène la prise de Sarajevo', qui, on s'en souvient, fut l'un des événements les plus marquants de la guerre de Bosnie-Herzégovine. Intitulé Robert Mitchum ne revient pas $^{2}$, le récit fait intervenir un matériau thématique peu employé dans la littérature de guerre: le tir sportif. L'intrigue se noue ainsi autour de deux protagonistes yougoslaves, surpris par l'imminence du blocus imposé par les forces serbes, alors même qu'ils se préparent aux Jeux Olympiques de Barcelone dans une discipline où ils excellent tous les deux : le tir à la carabine.

Devrait-on considérer le sport comme une métaphore de la guerre, lui prêtant son langage spécifique afin de "mettre sous les yeux», selon la formule de Paul Ricœur $(1975,263)$, une certaine idée du conflit ? C’est ce que justifieraient les analogies, dans le livre d'Hatzfeld, entre champ de bataille et affrontement sportif. Faudrait-il, au contraire, voir dans le sport une forme d'antithèse à la logique guerrière, à l’image des deux figures d'athlètes - Marija

\footnotetext{
${ }^{1}$ Rappelons-le, le siège, déclenché en avril 1992, fut le prélude à des affrontements de grande envergure entre Serbes, Croates et Bosniaques (Musulmans) s'étendant dans toute la Bosnie-Herzégovine.

${ }^{2}$ Hatzfeld, Jean. Robert Mitchum ne revient pas. Paris: Gallimard, 2013. Dans le corps de l'article, les citations tirées de ce livre seront suivies du titre abrévié $(R M)$ et du numéro de la page.
} 
et Vahidin - qui ouvrent le récit et chez qui la performance sportive paraît transcender les évènements de la guerre ${ }^{3}$ ?

Comme l'annoncent ces questions, notre lecture du roman voudrait relever en quoi le dispositif «sportif» mis en place par l'auteur permet de témoigner sur la guerre de Bosnie-Herzégovine en la situant à la confluence entre violence et non-violence, combat juste et injuste. Dans ce but, nous avancerons l'hypothèse d'une double valence attachée au paradigme sportif dans le roman. Sous un certain angle, en effet, le sport est en conjonction avec la guerre. Il peut être vu comme un moyen de préparation au combat. Par la même occasion - et c'est d'autant plus vrai pour le tir sportif -, il appelle à pénétrer l'univers du militaire. Il peut donc déboucher sur une vision intime de son engagement. À l'extrême opposé, le sport s'énonce en complet divorce avec la guerre. Il se laisse alors appréhender à l'aune d'une violence enfin canalisée, rendue conforme à un programme qui n'a plus rien en commun avec la pratique guerrière proprement dite ${ }^{4}$. Le sport aurait dès lors évacué ce sur quoi la guerre repose : l'acte de tuer. Il serait l'expression d'un discours pacifiste, humaniste et même d'un idéal civilisateur (aujourd'hui, ce dernier tend d'ailleurs à refaire surface avec l'avènement des jeux de combats en ligne, où l'affrontement est sans conséquence véritable).

Nous étudierons donc les procédés par lesquels Hatzfeld exploite cette dualité des rapports entre sport et guerre. Par là, il s'agira d'envisager comment il ne témoigne pas tant du combat dans ses dimensions stratégiques et tactiques, mais plutôt de la façon dont les individus vivent la guerre, au quotidien. Son roman illustre de la sorte le parcours de deux athlètes olympiques qui entendent rester à l'écart du conflit, mais qui, malgré eux, vont être progressivement entrainés dans l'effort de guerre, endossant alors les compromissions qu'elle impose.

\section{Sport, guerre et journalisme}

Afin de saisir tout l'enjeu de cette coïncidence entre sport et guerre, il nous paraît essentiel de situer d'abord le texte à l'étude à la confluence entre discours et société. C'est que le choix d'une perspective sportive pour

${ }^{3}$ Le roman s'ouvre sur une scène in medias res dans laquelle les deux athlètes - l'un étant Musulman bosniaque et l'autre d'origine serbe (faisant dès lors écho à la dimension raciale du conflit, mais pour en souligner l'impertinence) - courent côte à côte, en portant «les survêtements rouge, bleu, blanc de l'équipe yougoslave» (là aussi, non sans sous-entendu). Leur effort physique fait oublier la guerre, à la manière d'une "transpiration » dissipant l'évocation des horreurs qu'elle engendre. La narration coïncide avec ce parti pris, la litote étant utilisée pour mettre en sourdine ce qui apparaît alors comme un écho lointain des affrontements.

4 À ce propos, Luc Robène fait la remarque suivante, qui annonce tout l'enjeu de cette rencontre, sur le plan discursif, entre sport et guerre : «Le sport, la compétition sportive, marque l'avènement d'une forme d'utopie: la perspective idéalisée d'une guerre jouée, d'une guerre pacifiée en quelque sorte, s'inscrivant dans une société apaisée, via un ensemble de dispositifs spécifiques (olympisme, grands jeux, grandes compétitions internationales, standardisation des formes de rencontre et des normes de l'affrontement) qui permettent de canaliser, de réguler jusqu'aux manifestations sociales les plus agressives. » (2012, 9). 
«fictionnaliser» la guerre n'a rien de fortuit : il peut s'interpréter comme une prise de position idéologique à l'égard d'autres discours avec lesquels il interagit (Zima, 2000) ; en l'occurrence, le discours journalistique. En effet, Hatzfeld était, dès 1973, journaliste au quotidien français Libération, écrivant principalement sur le sport, avant de devenir correspondant de guerre d'abord au Moyen-Orient puis en Europe centrale. Selon une conception propre à Pierre Bourdieu $(1980,88)$ - qui, rappelons-le, a développé la notion d’habitus -, on pourrait supposer qu'en tant qu'ancien journaliste sportif, l'auteur a acquis certains modes de perception et d'appréciation qui l'ont prédisposé à appréhender les réalités du conflit bosniaque à partir d'un terrain aussi singulier que celui du tir sportif (inclus au programme des Jeux Olympiques depuis l'année 1896). La métaphore du sport, au sein d'un univers fictionnel dominé par l'affrontement armé, serait alors conçue comme un stratagème cognitif pour témoigner d'une réalité de la guerre que l'auteur n'a que partiellement connue, n'ayant lui-même pas directement pris part au conflit. C'est certainement vrai, mais cela n'explique pas tout.

Il nous faut nous attarder sur un autre roman de l'ancien journaliste, $\mathrm{La}$ Ligne de flottaison (2005), si l'on veut comprendre comment le récit sportif peut s'inscrire à la fois comme mode esthétique et cognitif pour dire la guerre et comme prise de position littéraire sur des questions testimoniales. La Ligne de flottaison met donc en scène un correspondant de guerre - dénommé Frédéric - qui n'a de cesse de revenir sur les causes qui l'ont conduit à choisir une telle profession, l'exposant non seulement au risque de périr, mais l'éloignant aussi des siens. Interrogé par ses collègues de travail et, par ailleurs, se questionnant soi-même sur ses propres pratiques, le récit donne lieu à un long débat sur le rôle du journaliste au sein de sa société et vis-à-vis des communautés en guerre qu'il côtoie; un rôle bien difficile à cerner à cause de la position en porte-à-faux du reporter de guerre :

On n'écrit pas sur la guerre pour ceux qui la vivent, répliqua Antoine. Ceux-là s'en fichent bien la plupart du temps, en tout cas sur le moment. Tu as l'air d'avoir été tétanisée parce que tu n'existais pas à leurs yeux, ça me semble une réaction plus qu'étrange. Comme si tu espérais une reconnaissance de leur part pour être avec eux... Au Kivu, en plus.

- Bon, d'accord, on ne doit envisager aucune complicité ou aucune compréhension entre eux et nous. Surtout là-bas. Mais quand même des gestes humains, des mots, non ? Le rejet absolu n'est pas vivable. Ils me regardent de loin. Ce silence. Ils m'avaient exclue, c'est ça, eh bien moi je fais avec. Ils se taisent, je me tais. Si une femme m'avait demandé ce que je faisais là, ou si elle m'avait demandé de l'argent, ou m'avait insultée, j’aurais sans doute sorti mon carnet. $(R M, 166)$.

Une polémique entre différentes conceptions du journalisme de guerre (en l'occurrence, la guerre du Kivu dans l'est du Congo) s'amorce à cette étape de la discussion, soulevant le problème de l'inefficacité des reporters présents sur le terrain. Ces derniers ayant l'impression de ne pas être du même univers que les populations locales forcées de subir la dureté du conflit (tandis qu'eux, 
ils peuvent repartir), ils éprouvent une mauvaise conscience qui renforce inévitablement leur distance toute voyeuriste. Ce n'est alors pas l'incapacité des journalistes à communiquer les faits dont ils sont les témoins directs qui est en cause, mais l'abîme qui existe entre le lecteur occidental pour qui ils écrivent et les civils, acteurs ou victimes du conflit. La réplique de Betty: «Mais quand même des gestes humains, des mots, non ? Le rejet absolu n'est pas vivable.» $(R M, 166)$ souligne ce qui, au fond, fait défaut au témoignage journalistique sur la guerre s'il ne veut pas rester qu'une parole creuse ou, selon l'expression latine, un flatus vocis: le signe d'une réelle proximité empathique entre le récepteur du discours médiatique et le civil qui se voit raconté.

À la lumière de cette digression (que nous fermons ici), nous pouvons maintenant mieux concevoir pourquoi le sport dans Robert Mitchum ne revient pas a, au-delà de ses fonctions esthétique et cognitive, une valeur de contre-discours: l'évocation du sport, ou plutôt du sport dans la guerre, favoriserait cette jonction à la fois empathique et réflexive qui fait défaut au discours journalistique. En d'autres termes, Hatzfeld essaye de créer les conditions d'un récit sur la guerre où le lecteur n'occuperait pas la place d'un spectateur passif ou d'un tiers (à connotation péjorative). La thématique athlétique a donc, dans son roman, une portée qui n'est pas, par exemple, celle de Georges Perec dans W ou le Souvenir d'enfance (1975) (récit dans lequel elle renvoie explicitement au système concentrationnaire). L'univers de la compétition sportive - sans doute à cause de sa charge affective et du sentiment d'étonnement qu'il génère dans le contexte d'une fiction de la guerre - conduit ainsi à établir certaines passerelles entre le lecteur, les personnages et les évènements représentés, au point que le regard du lecteur sur les évènements n'a plus rien de commun avec celui qui a par exemple dominé certains médias au moment de l'éclatement de la Yougoslavie ${ }^{5}$.

\section{Valence 1 : la guerre et le sport s'imbriquent}

L'interrelation entre le sport et la guerre se manifeste d'emblée du point de vue narratif. C'est que le tir sportif, pratiqué à l'échelle internationale par les deux protagonistes du récit, les prédestine très vite à occuper une fonction déterminante dans la guerre.

Rappelons brièvement l'intrigue : Marija et Vahidin, un couple mixte (la première est Serbe et le second, Bosniaque) qui n'est pas sans évoquer les amants Admira et Boško ${ }^{6}$, s'entrainent conjointement au tir en vue des Jeux de Barcelone quand, malgré eux, l'installation du siège de Sarajevo les sépare. Marija reste à Ilidža, un faubourg de Sarajevo devenu une zone serbe, tandis que Vahidin se voit confiné dans la partie musulmane de la ville, sous blocus. Ce dernier tente vainement de communiquer avec sa partenaire restée à Ilidža.

5 Pour un état des lieux sur la question des médias occidentaux lors des guerres en ex-Yougoslavie, on peut se référer au dossier de revue d’Éric Pédon et Jacques Walter (2002).

${ }^{6}$ Cette anecdote, devenue célèbre à Sarajevo, est évoquée au chap. XVII du roman à l'étude. Elle a aussi fait l'objet d'un film documentaire (Zaritsky, 1994). 
Alors qu'il espère justement lui faire passer une lettre en tirant parti des forces en présence sur le terrain, un certain Mirsad - un ami d'enfance qui dirige depuis peu une brigade anti-serbe -, le sollicite afin de mener à bien une mission extrêmement pointue que seul un champion du tir peut accomplir. Elle consiste à débouter des tireurs d'élite qui ont pris position sur les hauteurs de Sarajevo et visent en direction de la ville assiégée ( $R M, 46-47)$. Vahidin accepte, escomptant que cet accord le préservera du conflit et de ses implications (ce qui est bien sûr paradoxal). Un peu plus en avant ( $R M, 75-76)$, Marija accepte une proposition similaire de deux officiers serbes venus à sa rencontre, et qui lui demandent de prendre position dans un appartement de l'ancien village olympique de Dobrinja tout en tenant à distance des combattants bosniaques qui opèrent à proximité de l'aéroport.

Dans chacun des deux cas, on observe ainsi comment la pratique militaire s'inscrit dans la continuité directe de la pratique sportive. À la compétence des deux sportifs, qui justifie leur conscription, s'ajoutent des perspectives nouvelles qui tiennent, en partie du moins, à la consubstantialité des pratiques sportive et guerrière. Leur alliance respective avec l'un ou l'autre des camps antagonistes leur donne la possibilité de poursuivre leur entrainement aux Jeux et, corolairement, d'espérer pouvoir s'extraire d'un contexte politique de plus en plus tendu. Par ailleurs, s'agissant de Vahidin, une occasion inespérée se présente : celle de tenir entre ces mains un Dragunov, le fusil des tireurs d'élite russes.

En établissant les termes d'une relation métaphorique entre les sphères sportive et guerrière (autour de l'action du tireur qui sert à la fois de pivot au récit et de sème commun reliant le combat armé et l'affrontement sportif dans ses origines antiques), Hatzfeld peut alors problématiser la facilité avec laquelle le conflit s'empare des destinées individuelles, souvent de façon insidieuse. Ainsi, si, de prime abord, Marija et Vahidin acceptent sans trop de heurt de passer du tir olympique à l'engagement armé, c'est que leur nouveau statut de tireur d'élite n'est pas a priori en contradiction avec leurs valeurs sportives. Après tout, Vahidin n'accepte de prendre les armes contre les belligérants serbes que dans la mesure où il croit que ces derniers visent des civils sans défense ; autrement dit, parce qu'il y a eu agression:

- On a besoin de toi, Vahidin.

- De moi ? Pourquoi moi ? Écoute...

- Tu as bien vu que les Tchetniks placent des snipers en haut de Bistrik. De là, ils tirent sur la Baščaršija et Vratnik. Il y en a aussi au-dessus de Skenderija. D'en haut, ils visent ce qu'ils veulent, ils ont beaucoup tué déjà. Ils tirent les passants, et en particulier ceux qui marchent lentement, les vieux et les bonnes femmes avec des mômes. Ils ont abattu la gamine d'Esma tout à l'heure, sur Kovači, là, juste devant. (RM, 46).

Ce à quoi il est fait allusion dans cette réplique de Mirsad, c'est justement l'existence de codes de conduite de la guerre qui la rapproche d'une certaine éthique sportive (reposant aussi sur des codes). Ainsi les raisons 
alléguées pour engager le combat paraissent-elles pleinement justifiées ${ }^{7}$ : des civils sont massacrés; il y a donc eu provocation. Par ailleurs, on découvre que les moyens que Vahidin va utiliser pour combattre ses opposants serbes, les "Tchetniks », s'inscrivent dans une optique semblable au défi sportif (thème d'ailleurs largement développé dans le roman) : il les affrontera sur le même terrain qu'eux, où il sait qu'il pourrait tout aussi bien perdre l'avantage. Le personnage affiche par là un certain sens de la joute, de l'affrontement d'égal à égal, qui est en directe continuité avec les disputes sportives qu'il engage au niveau olympique. Au contraire, ses adversaires profitent de leur supériorité tactique pour semer la panique dans les rues de Sarajevo.

Mais ce n'est pas tout. Hatzfeld renforce cette coïncidence entre le duel sportif et une certaine pratique du combat armé en mêlant les deux registres sportif et militaire - sur le plan stylistique, comme l'illustre cet épisode dans lequel Vahidin, posté derrière une fenêtre d'immeuble, est à l'affut d'un tireur embusqué :

Plusieurs autres détonations suivirent. Une des fenêtres de la maison marron était grande ouverte. Le sniper se sentait assez en confiance pour laisser dépasser le bout du canon. Vahidin se concentra sur les infimes remous de l'air qui trahissaient le sniper près de l'encadrement. Il le laissa tirer plusieurs balles, le temps de se représenter mentalement sa silhouette. Il chercha son aplomb, bloqua son bassin en antéversion, enroula son épaule sur la crosse, amena son index sur la détente. Il contracta son dos, le relâcha et tassa son cou pour avoir la fenêtre dans la mire. Il s'immobilisa en apnée et appuya sur la détente, trois fois. $(R M, 52)$.

Dans cet extrait, la sémantique sportive se lit au travers d'une scène de combat, dans laquelle le narrateur, au lieu de relater le simple fait de donner la mort (de façon laconique), décrit la gestuelle du tireur à la manière d'un commentaire de journaliste sportif. Le narrateur décompose ainsi la manœuvre en ses différentes phases - «Vahidin se concentra sur les infimes remous de l'air»; « [i]l chercha son aplomb»; etc. -, fait appel à un savoir spécialisé et se projette dans l'esprit du tireur afin de décrire la préparation mentale. Dès lors, la manière de rendre compte du combat armé en est fortement influencée. Celui-ci paraît d'autant plus humain qu'il fait appel à des procédures bien réglées, une maîtrise disciplinaire. Il est un art, une technique, définissant alors un produit de la civilisation (dans l'acception universaliste que la notion a pu acquérir en conjonction avec l'idée de progrès), et non pas un acte violent, barbare, arbitraire et transgressif, comme il le deviendra progressivement dans la suite de l'histoire.

S'agissant de Marija, les raisons alléguées pour faire la guerre - le jus ad bellum et le jus in bello - sont d'autant plus conformes à l'entendement sportif que les officiers qu'elle rencontre ne l'oblige aucunement, en réalité, à s'impliquer directement dans les combats : «Alors, toi, on t'installe dans un

\footnotetext{
7 Dans son étude intitulée Guerres justes et injustes, Michael Walzer (1977) utilise à ce propos l'expression «jus ad bellum ».
} 
appartement à Dobrinja, et on te demande de les [les "Musulmans"] tenir à distance. Tu ne risques rien. Tu ne les combats pas, tu les tiens au bout de ta lunette et tu leur rappelles les bonnes manières. [...] Ça ne te mouille pas. » (RM, 76). Sa mission s'inscrivant dans le cadre d'une trêve entre Serbes et Bosniaques, occasionnée par le pèlerinage dans la ville de Sarajevo d'une célèbre diva américaine, elle n'aura donc pas à tuer. Le même ton prédomine dans les descriptions de la préparation à l'arme ; comme pour le protagoniste masculin, la finalité de la mort cède la place à un registre qui mêle technicité et sensualité (pour ne pas dire érotisme) :

Marija attendit son immobilisation en bout de piste pour attraper son fusil et stabilisa ses pieds parallèles au bord de la fenêtre. Elle remua ses orteils, l'un après l'autre, pour les décrisper, pressa le sol de ses talons et étala son poids sur la plante du pied. Elle fléchit les jambes, un peu plus que d'habitude, se dit-elle. Elle cala le bec de la crosse contre son sein, décontracta son bras avant de trouver, sur le dos de ses doigts repliés, l'appui sous le canon. Elle soupira de se sentir si relâchée et commença à scruter les fenêtres alentour. $(R M, 103)$.

Jusqu'ici, pratique sportive et pratique guerrière ne semblent pas antinomiques: qu'il s'agisse du combat ou de l'affrontement sportif, les normes d'action et les soubassements moraux restent similaires. Hatzfeld, d'ailleurs, n'a de cesse de faire coïncider les deux isotopies en établissant des jeux de miroirs, des échos, à divers endroits du récit. Pour sa part, le geste de donner la mort est mis en sourdine.

\section{Valence 2 : des jeux de non-coïncidence}

Malgré les rapports de concomitance entre sport et guerre, par lesquels la lutte armée apparait comme un affrontement entre professionnels et selon des codes précis, Hatzfeld entend aussi souligner à quel point les enjeux fondamentaux du sport et de la guerre diffèrent. Cette distinction entre valeurs sportives et valeurs guerrières permet au romancier de questionner l'impact des conflits sur ceux qui les vivent comme une contrainte extérieure, voire une hétéronomie $^{8}$, parfois sans en saisir la logique? ${ }^{9}$. Il s'agit alors d'interroger le vécu d'individus qui, à la manière du lecteur hors frontières (dont le texte reflète aussi la perspective ${ }^{10}$ ), ont un rapport distant ou décalé avec les événements même s'ils se trouvent être au cœur de l'horreur.

\footnotetext{
${ }^{8}$ Dans le sens où leurs croyances, leur perception et leur conduite seraient guidées par certains contours de la guerre.

9 La décision de situer le décor romanesque à Sarajevo même, ville parfois appelée «la cosmopolite » à cause de son caractère transculturel ainsi que de son foisonnement artistique et culturel, est, à ce titre, bien emblématique.

${ }^{10}$ Les limites de cet article ne nous permettent malheureusement pas de développer cet aspect. Toutefois, il faut avoir connaissance du fait que le roman intègre une intrigue secondaire, assez lâche, mais qui constitue une mise en abîme du récit en tant que témoignage de la guerre. L'histoire relate le parcours de trois journalistes français prenant le chemin du front qui, une fois arrivés là-bas, adoptent d'abord un regard voyeuriste sur les atrocités de la guerre, en miroir bien sûr à celui de leurs lecteurs distants.
} 
Dès les premières pages, le roman invite ainsi à considérer le déchirement de Marija et Vahidin entre deux conceptions opposées des réalités sociales, qui ont toujours été présentes de manière insidieuse, mais qui vont cette fois les prendre au dépourvu. La première conception se lit à travers les changements dans l'environnement immédiat des deux protagonistes, notamment en ce qui concerne l'attitude de leurs pairs : il ne peut y avoir d'entente possible entre Serbes et Bosniaques. La séparation du couple, sous la pression des circonstances externes, devient ainsi une allégorie du clivage Serbes/Bosniaques: même les moins enclins à admettre la nécessité de la division raciale doivent la subir. Or, c'est par l'entremise de la compétition sportive et, plus particulièrement, de la préparation aux Jeux de l'été 1992 que les deux protagonistes vont perpétuer un lien psychique au-delà de la séparation de fait.

Le récit, à travers les choix narratifs dont il témoigne, se charge dès lors de véhiculer des valeurs morales et une perception du monde en relation étroite avec l'univers du sport moderne. En effet, un certain universalisme sportif, qui a son expression la plus directe dans la vocation internationale et pacifique du Mouvement olympique ${ }^{11}$, trouve écho dans la conscience des deux protagonistes, qui parviennent, par là, à ne pas céder totalement à l'esprit de la guerre. À ce sujet, toutes les séquences narratives où l'on voit Vahidin à l'affût des tireurs d'élite serbes jouent sur un renversement assez caractéristique. À chaque reprise, en effet, la narration brise l'effet d'attente d'une cible ennemie pour montrer le personnage qui anticipe au contraire la préparation de sa partenaire - «Serbe » (lui-même étant, depuis quelques mois à peine, placé sous l'égide d'une autre nation) - pour les Jeux Olympiques. De ce fait, l'enjeu du suspense est complètement décalé :

Il [Vahidin, à l'affût d'un tireur] s'inquiéta pour Marija, car il savait qu'elle craignait la solitude. Dans sa lettre, elle lui écrivait qu'elle avait récupéré Mitchum, mais ne disait rien sur le Klub. Sont-ils partis en stage au Monténégro ? Respecte-t-elle le planning mis au point par le coach ? Vahidin en doutait car, livrée à elle-même, Marija a tendance à sous-estimer le travail de fond. Elle a une chance énorme de gagner aux Jeux, Vahidin en était plus convaincu qu'elle. Sa technique baroque fait jaser, mais sa complicité ahurissante avec la carabine la légitime. (RM, 55).

À ce stade de l'intrigue, Vahidin ignore que Marija puisse, comme lui, s'engager à titre de tireur embusqué dans le camp adverse et, par là, constituer une menace potentielle. Cette confrontation aura d'ailleurs lieu plus tard, mais de façon inconsciente et détournée: Vahidin prendra pour cible la cantatrice Carroll Del Rio, sans se douter que Marija, postée à un immeuble adjacent, sera elle-même accusée par l'opinion publique de l'attentat (simulé) commis par lui. Toutefois, tirant parti de ce décalage dans l'ordre du savoir, le récit joue sur ce paradoxe par lequel deux adversaires sur le plan militaire puissent

${ }^{11}$ La Charte olympique s'en fait d'ailleurs l'écho dans l'énoncé de la mission et du rôle du Comité International Olympique (CIO 2013). 
s'encourager dans la pratique des armes - à l'échelle sportive. De ce fait, leurs interrogations sur leurs chances réciproques de remporter la victoire aux Jeux visent, implicitement, à établir une distinction entre les enjeux du sport et ceux de la guerre. C'est toute la notion de fair play qui est thématisée ici, le sport incarnant des valeurs sociales, se référant par ailleurs à un code d'honneur et à un état d'esprit qui n'ont pas la même portée sur le terrain des opérations, où, par exemple, des civils peuvent être tués ${ }^{12}$.

De manière générale, l'ensemble des partis pris esthétiques, dans le roman, vient appuyer cette démarcation entre pratique sportive et pratique guerrière. On l'a vu, le paradigme sportif convoque les valeurs d'un savoir faire, alors que la rhétorique guerrière repose sur des critères d'appartenance qui sont vus comme l'envers de tout savoir. Toute la mise en scène de la technique sportive n'est d'ailleurs pas sans rappeler, historiquement, l'apologie du progrès, des Lumières aux avancées de la physique moderne, sans oublier les anciens empires coloniaux et leur projet civilisateur. La vision du corps athlétique - celle d'un corps tendu, dynamique et précis - ne traduit-elle d'ailleurs pas, à titre de métonymie, le perfectionnement humain, comme l'avait bien compris Leni Riefenstahl (1938) dans ses films de propagande? Sur un autre plan, la métaphore amoureuse vient conforter les investissements sportifs, quand la guerre conduit à une série de trahisons, qui ont aussi leur composante affective: Vahidin est séduit par une Bosniaque musulmane, Edina, ce qui provoque la consternation de Marija et décide, dans une certaine mesure, de sa participation dans le conflit.

Il faut enfin évoquer la dimension collective du sport qui s'inscrit de manière décisive dans le roman. Le sport, en effet, n'apparaît pas seulement comme une pratique individuelle; il implique également une communauté avec ses institutions, ses sphères d'influences, sa «vitrine» sur l'extérieur. Marija est ainsi exclue des Jeux par la communauté internationale à cause de son rôle dans l'armée serbe et des soupçons d'attentat qui pèsent sur elle. Les mêmes circonstances surviennent lorsque les deux protagonistes sportifs, à nouveau réunis, sont surpris aux Jeux Olympiques de Sydney par une interprète qui fait diffuser le cliché de leur rencontre dans la presse internationale. On voit bien ici de quelle façon le sport, dans sa conjonction avec la société du spectacle, acquiert une dimension politique qui vient influer sur la guerre elle-même. À ce stade-ci, un nouvel enjeu se profile : comment le sport, dans sa dimension spectaculaire, prolonge-t-il l'entreprise de destruction amorcée par la guerre? Une intertextualité s'établit dès lors entre le récit

${ }^{12}$ La manière dont le concept de fair play est aujourd'hui saisi dépasse généralement le cadre strictement sportif, afin de démontrer sa force d'exemplarité à l'échelle des collectivités et du rapport entre les Nations. L'UNESCO s'en fait d'ailleurs l'écho dans sa Charte internationale de l'éducation physique et du sport (1978) : «Au niveau de la communauté, [l'éducation physique et le sport] enrichissent les rapports sociaux et développent l'esprit sportif (fair play qui, au-delà du sport lui-même, est indispensable à la vie en société) » (33). Une abondante bibliographie sur le fair play et ses relations avec l'éthique de la non-violence est également disponible sur le site Web du Mouvement Européen pour le Fair-play (2014). 
sportif et le spectacle antique. C'est que deux conceptions du sport sont en jeu dans le roman: d'une part, le sport comme exploit, dans sa dimension «civilisatrice » et, d'autre part, le sport comme spectacle, qui, lui, renoue par certains aspects avec la cruauté de la guerre.

\section{Conclusion}

On remarque donc à quel point le témoignage littéraire de la guerre peut tirer profit de la mise en rapport entre plusieurs réalités et, a fortiori, entre plusieurs domaines de connaissance. L'idée, en soi, n'est pas nouvelle. On sait ainsi que les romanciers de la Shoah ont souvent eu recours à des matériaux variés pour témoigner de l'expérience concentrationnaire, pour laquelle il n'existait pas de langage spécifique. Plus tard, suite aux événements du génocide rwandais, une certaine littérature africaine a repoussé les limites de ce qui avait été amorcé par les romanciers de la Shoah. Cela a donné lieu à un courant d'études critiques gravitant autour des notions d'interdiscours, ou encore de transculture. La littérature des violences extrêmes semble ainsi être très élastique ; propice à l'intégration de matériaux hétérogènes pour créer son propre discours. Mais qu'en est-il exactement du témoignage de la guerre? Cette question se pose, car, à la différence du génocide par exemple, la guerre convoque une forme «assumée » de violence ; une violence impliquant a priori qu'elle soit exprimable, qu'elle ait donc son langage propre. L'exemple de Jean Hatzfeld montre bien que si la métaphore du sport possède son efficacité pour dire la guerre, c'est qu'elle implique une réalité qui recoupe au moins partiellement celle de la guerre. Les enjeux du sport moderne, au travers de compétitions internationales telles que les rencontres olympiques, ne sont pas non plus sans rapport avec les débats qui agitent le monde de la guerre moderne.

\section{Bibliographie}

Bertrand, Denis. Précis de sémiotique littéraire. Paris : Nathan, 2000.

Bourdieu, Pierre. Le Sens pratique. Paris : Minuit, 1980.

CIO. Charte olympique: état en vigueur au 9 septembre 2013. Lausanne: Comité International Olympique, 2013.

Hatzfeld, Jean. La Ligne de flottaison. Paris : Seuil, 2005.

Hatzfeld, Jean. Robert Mitchum ne revient pas. Paris : Gallimard, 2013.

Mouvement Européen pour le Fair-play (EFPM). "Fair Play Bibliography». [En ligne]. Mis en ligne le 23 septembre 2014. URL: http://www.fairplayeur.com/?page_id=60. (Consulté le 14 août 2015).

Pédon, Éric et Jacques Walter (dir.), Questions de communication, «Les médias et les guerres en ex-Yougoslavie » $\mathrm{N}^{\circ} 1$ (2002). [En ligne]. URL :http://questionsde communication.revues.org/6455. (Consulté le 14 août 2015).

Perec, Georges. W ou le souvenir d'enfance. Paris : Denoël, 1975.

Ricœur, Paul. La métaphore vive. Paris : Seuil, 1975.

Riefensthal, Leni. Olympia. 1938. 
Robène, Luc. Le Sport et la guerre : XIX'e et XXe siècles. Rennes : Presses universitaires de Rennes, 2012.

UNESCO. Charte internationale de l'éducation physique et du sport. Paris: Organisation des Nations Unies pour l'éducation, la science et la culture, 1978.

Walzer, Michael. Just and unjust wars : a moral argument with bistorical illustrations [Guerres justes et injustes: argumentation morale avec exemples historiques]. New York: Basic Books, 1977.

Zaritsky, John. Romeo and Juliet in Sarajevo [Roméo et Juliette à Sarajevo]. 1994.

Zima, Pierre V. Pour une sociologie du texte littéraire. Paris : L'Harmattan, 2000 [1978]. 
